\title{
APPROXIMATION OF ENTIRE FUNCTIONS OVER CARATHEODORY DOMAINS
}

\author{
G.P. Kapoor and A, Nautiyal
}

Let $D$ be a domain bounded by a Jordan curve. For $1 \leq p \leq \infty$, let ${ }_{L}^{p}(D)$ be the class of all functions $f$ holomorphic in $D$ such that $\|f\|_{D, P}=\left((1 / A) \iint_{D}|f(z)|^{p} d x d y\right)^{1 / p}<\infty$, where $A$ is the area of $D$. For $f \in L^{p}(D)$, set

$$
E_{n}^{p}(f)=\inf _{g \in \pi_{n}}\|f-g\|_{D, p}
$$

$\pi_{n}$ consists of all polynomials of degree at most $n$. Recently, Andre Giroux (J. Approx. Theory 28 (1980), 45-53) has obtained necessary and sufficient conditions, in terms of the rate of decrease of the approximation error $E_{n}^{p}(f)$, such that $f \in L^{p}(D), 2 \leq p \leq \infty$, has an analytic continuation as an entire function having finite order and finite type. In the present paper we have considered the approximation error (*) on a Carathéodory domain and have extended the results of Giroux for the case $1 \leq p<2$.

\section{Introduction}

Let $B$ denote a Carathéodory domain, that is, a bounded simply

Received 29 September 1981. 
connected domain such that the boundary of $B$ coincides with the boundary of the domain lying in the complement of the closure of $B$ and containing the point $\infty$. In particular, a domain bounded by a Jordan curve is a Carathéodory domain. Let $L^{p}(B), I \leq p \leq \infty$, be the class of all functions $f$ holomorphic on $B$ and satisfying

$$
\left.\|f\|_{B, p}=\iiint_{B}|f(z)|^{p} d x d y\right)^{1 / p}<\infty,
$$

where the last inequality is understood to be $\sup _{z \in B}|f(z)|<\infty$ for $p=\infty$. Then $\|\cdot\|_{B, p}$ is called the ${ }_{L}^{p}$-norm on ${ }_{L}^{p}(B)$. For $f \in L^{p}(B)$, we define $E_{n}^{p}(f)$, the error in approximating the function $f$ by polynomials of degree at most $n$ in $L^{p}$-norm, as

$$
E_{n}^{p}(f) \equiv E_{n}^{p}(f, B)=\inf _{g \in \pi_{n}}\|f-g\|_{B, p}, n=0,1,2, \ldots,
$$

where $\pi_{n}$ consists of all polynomials of degree at most $n$.

We prove

THEOREM 1. Let $f \in L^{p}(B), 1 \leq p \leq \infty$. Then $f$ is the restriction to $B$ of an entire function, if and only if,

$$
\lim _{n \rightarrow \infty}\left(E_{n}^{p}(f)\right]^{1 / n}=0 \text {. }
$$

For the case $p=\infty$, it is sufficient to assume that $f$ is continuous on $B$.

THEOREM 2. Let $f \in L^{p}(B), 1 \leq p \leq \infty$. Then $f$ is the restriction to $B$ of an entire function of finite order $\rho$, if and only if,

$$
\underset{n \rightarrow \infty}{\lim \sup }\left((n \log n) /\left(-\log E_{n}^{p}(f)\right)\right)=\rho
$$

and, if $\rho>0$, of nonzero finite type $T$, if and only if, 


$$
\underset{n \rightarrow \infty}{\lim \sup } n\left(E_{n}^{p}(f)\right)^{\rho / n}=e \rho d^{\rho_{T}}
$$

where $d$ is the transfinite diameter of the closure of $B$. For the case $p=\infty$, it is enough to assume that $f$ is continuous on $B$.

REMARKS. (i) Results of the nature of Theorems 1 and 2 , in $L^{\infty}$-norm, have been extensively studied by various workers (for example, Bernstein [1, p. '113], [5, pp. 76-78], Varga [10], Shah [8], Kapoor and Nautiyal [4], Winiarski [11]).

(ii) For $p=2$ and $B=\{z:|z|<1\}$, Theorems 1 and 2 are due to Reddy [7].

(iii) Theorems 1 and 2 extend and generalize the results of Giroux [3], obtained for the case $2 \leq p \leq \infty$ with $B$ as a domain bounded by a Jordan curve.

\section{Proofs of the theorems}

Let $B^{*}$ be the component of the complement of $\bar{B}$, the closure of $B$, that contains the point $\infty$. Set $B_{r}=\{z:|\varphi(z)|=r\}, r>I$, where the function $w=\varphi(z)$ maps $B^{*}$ conformally onto $|w|>1$ such that $\varphi(\infty)=\infty$ and $\varphi^{\prime}(\infty)>0$.

We need the following lemmas.

LEMMA 1 ([11, Lemma 3.1]). The order $\rho$ of an entire function $f(z)$ is given by

$$
\rho=\lim \sup _{r \rightarrow \infty}(\log \log \bar{M}(r, f)) /(\log r)
$$

and, if $0<\rho<\infty$, the type $T$ of $f(z)$ is given by

$$
T d^{\rho}=\underset{r \rightarrow \infty}{\lim \sup }(\log \bar{M}(r, f)) / r^{\rho}
$$

where $d$ is the transfinite diameter of $\bar{B}$ and

$$
M(r, f)=\max _{z \in B_{r}}|f(z)| .
$$

LEMMA 2. Let $f \in L^{p}(B), 1 \leq p<\infty$, be the restriction to $B$ of an entire function and let $r^{\prime}(>1)$ be given. Then, for all $r>2 r^{\prime}$ 
and all sufficiently large values of $n$, we have

$$
E_{n}^{p}(f) \leq K \bar{M}(r, f)\left(r^{\prime} / r\right)^{n}
$$

where $K$ is a constant independent of $n$ and $r$.

Proof. Since $f(z)$ is entire, there exists a sequence of polynomials $\left\{Q_{n}\right\}, Q_{n}$ being of degree at most $n$, such that

$$
\left|f(z)-Q_{n}(z)\right| \leq(3 / 2) \bar{M}(r, f) \frac{\left(r^{\prime} / r\right)^{n+1}}{1-\left(r^{\prime} / r\right)}, z \in \bar{B},
$$

for all $r>p^{\prime}$ and all sufficiently large values of $n$ ([6, p. 114]).

It follows from the definition of ${ }_{n}^{p}(f)$, since $Q_{n} \in \pi_{n}$, that

$$
E_{n}^{p}(f) \leq\left\|f-Q_{n}\right\|_{B, p} \leq A^{I / p} \max _{z \in \bar{B}}\left|f(z)-Q_{n}(z)\right|
$$

where $A$ is the area of $B$. The lemma now follows from (2.1) and $(2,2)$.

Proof of Theorem 1. If $f \in L^{p}(B), 1 \leq p<\infty$, is entire, it follows from Lemma 2 that $\underset{n \rightarrow \infty}{\lim \sup _{n \rightarrow \infty}}\left(E_{n}^{p}(f)\right)^{1 / n} \leq r^{\prime / r}$ for all $r>2 r^{\prime}$. Letting $r \rightarrow \infty$, we get

$$
\lim _{n \rightarrow \infty}\left(E_{n}^{p}(f)\right)^{1 / n}=0
$$

This proves the necessity part of the theorem for $1 \leq p<\infty$.

Now, let $z_{0} \in B$ and let $R>0$ be such that $D_{R}=\left\{z:\left|z-z_{0}\right| \leq R\right\}$

is contained in $B$. If $f \in L^{p}(B), 1 \leq p<\infty$, then $f(z)$ is holomorphic on $D_{R}$ and has the following Taylor expansion

$$
f(z)=\sum_{n=0}^{\infty} a_{n}\left(z-z_{0}\right)^{n}
$$

in $D_{R}$, where the $a_{n}^{\prime}$ 's are given by

$$
\frac{\pi R^{2(n+1)}}{n+1} a_{n}=\iint_{D_{R}} f(z) \overline{\left(z-z_{0}\right)^{n}} d x d y
$$


Thus, for any $g \in \pi_{n-1}$, we have

$$
\begin{aligned}
\frac{\pi R^{2(n+1)}}{n+1}\left|a_{n}\right| & =\left|\iint_{D_{R}}(f(z)-g(z)) \overline{\left(z-z_{0}\right)^{n}} d x d y\right| \\
& \leq R^{n}\|f-g\|_{B, 1} .
\end{aligned}
$$

Now, using Hölder's inequality, the above relation gives that

$$
\frac{\pi R^{n+2}}{n+1}\left|a_{n}\right| \leq A^{q}\|f-g\|_{B, p}
$$

where $A$ is the area of $B$ and $q=1-1 / p$. Since the above relation holds for any $g \in \pi_{n-1}$, we have

$$
\frac{\pi R^{n+2}}{n+1}\left|a_{n}\right| \leq A^{q} E_{n-1}^{p}(f)
$$

If, for $f \in L^{P}(B), I \leq p<\infty$, equation (1.1) holds, then it follows, from (2.4), that

$$
\lim _{n \rightarrow \infty}\left|a_{n}\right|^{1 / n}=0
$$

and so, (2.3) gives that $f(z)$ is an entire function. This proves the sufficiency part of the theorem for $1 \leq p<\infty$.

The theorem is thus proved for $1 \leq p<\infty$. For the case $p=\infty$, the theorem is essentially due to Winiarski [11].

Proof of Theorem 2. (i) First, let $f(z)$ be an entire function. Then ([9, p. 273]), for all finite $z$,

$$
f(z)=\sum_{n=0}^{\infty} b_{n} p_{n}(z)
$$

where $\left\{p_{n}\right\}_{n=0}^{\infty}$ is a sequence of polynomials, $p_{n}$ being of degree $n$, such that

$$
\iint_{B} p_{n}(z) \overline{p_{m}(z)} d x d y=\delta_{m}^{n}, \quad b_{n}=\iint_{B} f(z) \overline{p_{n}(z)} d x d y
$$

$\delta_{m}^{n}=1$ for $m=n$ and $\delta_{m}^{n}=0$ otherwise. It is also known [9, p. 272] 
that, given $r_{*}>1$, we have

$$
\max _{z \in \bar{B}}\left|p_{n}(z)\right| \leq C_{*}^{n}, n=1,2, \ldots,
$$

where $C$ is a constant independent of $n$.

From (2.6) and (2.7), for any $g \in \pi_{n-1}, n \geq 1$, we obtain

$$
\left|b_{n}\right|=\left|\iint_{B}(f(z)-g(z)) \overline{p_{n}(z)} d x d y\right| \leq C r_{*}^{n}\|f-g\|_{B, I} .
$$

On applying Holder's inequality, (2.8) gives

$$
\left|b_{n}\right| / r_{*}^{n} \leq C A^{q}\|f-g\|_{B, q}, \quad 1 \leq p<\infty,
$$

where $A$ is the area of $B$ and $q=1-1 / p$. Since the above relation holds for any $g \in \pi_{n-1}$, we have

$$
\left|b_{n}\right| / r_{*}^{n} \leq C A^{q} E_{n-1}^{p}(f), 1 \leq p<\infty .
$$

Now, using (2.5) and (2.7) and applying Bernstein's inequality [6, p. 112] to each term of the series $\sum_{n=0}^{\infty} b_{n} p_{n}(z)$, we obtain

$$
|f(z)| \leq\left|b_{0}\right|+C \sum_{n=1}^{\infty}\left|b_{n}\right|\left(r r_{*}\right)^{n}, z \in B_{r}
$$

The above relation, in view of (2.9), gives that

(2.10) $\bar{M}(r, f) \leq\left|b_{0}\right|+C^{2} A^{q} \sum_{n=1}^{\infty} E_{n-1}^{p}(f)\left(r r_{*}^{2}\right)^{n}, 1 \leq p<\infty$.

Set $f_{p}(z)=\sum_{n=0}^{\infty} E_{n}^{p}(f) z^{n}, 1 \leq p<\infty$. By Theorem 1, $f_{p}(z)$ is an entire function. Further, (2.10) gives that

$$
\bar{M}(r, f) \leq\left|b_{0}\right|+c^{2} A^{q} r r_{*}^{2} M\left(r_{*}^{2}, f_{p}\right) .
$$

In view of Lemma 1, from (2.11) we obtain

$$
\rho \leq \rho_{p}
$$


where $\rho$ is the order of $f(z)$ and $\rho_{p}$ is the order of $f_{p}(z)$.

On the other hand, by Lemma 2, we get

$$
\begin{aligned}
M\left(r / r^{\prime}, f_{p}\right) & \leq P(r)+K \bar{M}(r+1, f) \sum_{n=0}^{\infty}(r /(p+1))^{n} \\
& =P(r)+K(r+1) \bar{M}(r+1, f)
\end{aligned}
$$

where $P(r)$ is a polynomial. From (2.13) and Lemma 1, we have

$$
\rho_{p} \leq \rho
$$

Combining (2.12) and (2.14) we get $\rho_{p}=\rho$. Thus, applying the formula expressing the order of an entire function in terms of its Taylor coefficients $[2, \mathrm{p} .9]$ to the function $f_{p}(z)$, it follows that the order $\rho$ of $f(z)$ is given by (1.2).

(ii) If, for $f \in L^{p}(B), 1 \leq p<\infty$, the limit superior on the left hand side of (1.2) is finite, it follows that $\lim _{n \rightarrow \infty}\left(E_{n}^{p}(f)\right)^{1 / n}=0$. Hence, by Theorem 1, $f(z)$ is entire. From (i) we now get that the order $\rho$ of $f(z)$ is given by (1.2).

(iii) Let $f(z)$ be an entire function of order $\rho, 0<\rho<\infty$, and type $T$. Then, using (2.11), (2.13) and Lemma 1, we get

$$
T d^{\rho} \leq r_{*}^{2 \rho} T_{p}, \quad T_{p} /\left(r^{\prime}\right)^{\rho} \leq T d^{\rho}
$$

where $T_{p}$ is the type of the entire function $f_{p}(z)$. Since $r_{*}>1$ and $p^{\prime}>1$ are arbitrary, we get $T_{p}=T$. Thus, applying the formula expressing the type of an entire function in terms of its Taylor coefficients $[2, \mathrm{p} .11]$ to the function $f_{p}(z)$, it follows that the type $T$ of $f(z)$ is given by (1.3).

(iv) If, for $f \in L^{p}(B), 1 \leq p<\infty$, the limit superior on the left hand side of (1.3) is nonzero finite, it follows that

$$
\underset{n \rightarrow \infty}{\lim \sup _{n}}\left\{(n \log n) /\left(-\log E_{n}^{p}(f)\right)\right)=\rho \text {. }
$$

Hence, by part (ii), $f(z)$ is an entire function of order $\rho$. From part 
(iii), we now get that the type $T$ of $f(z)$ is given by (1.3). For $1 \leq p<\infty$, the theorem now follows from parts (i) to (iv) above. For $p=\infty$, the theorem is essentially due to Winiarski [11].

\section{References}

[1] Serge Bernstein, Leọons sur les propriétés extrémales et la meizleure approximation des fonctions analytiques d'une variable réelle (Gauthier-Villars, Paris, 1926).

[2] Ralph Philip Boas, Jr., Entire functions (Pure and Applied Mathematics, 5. Academic Press, New York, 1954).

[3] Andrê Giroux, "Approximation of entire functions over bounded domains", J. Approx. Theory 28 (1980), 45-53.

[4] G.P. Kapoor and A. Nautiyal, "Polynomial approximation of an entire function of slow growth", J. Approx. Theory 32 (1981), 64-75.

[5] G.G. Lorentz, Approximation of functions (Holt, Rinehart and Winston, New York, Chicago, London, 1966).

[6] A.l. Markushevich, Theory of functions of a complex variable. Vol. III, revised English edition (translated and edited by Richard A. Silverman. Prentice-Hall, Englewood Cliffs, New Jersey, 1967).

[7] A.R. Reddy, "A contribution to best approximation in $L^{2}$ norm", $J$. Approx. Theory 11 (1974), 110-117.

[8] S.M. Shah, "Polynomial approximation of an entire function and generalized orders", J. Approx. Theory 19 (1977), 315-324.

[9] V.I. Smirnov and N.A. Lebedev, Functions of a complex variable: constructive theory (translated by Scripta Technica. MIT Press, Cambridge, Massachusetts, 1968).

[10] Richard S. Varga, "On an extension of a result of S.N. Bernstein", $J$. Approx. Theory 1 (1968), 176-179. 
[11] T. Winiarski, "Approximation and interpolation of entire functions", Ann. Polon. Math. 23 (1970), 259-273.

Department, of Mathematics,

Indian Institute of Technology,

Kanpur 208016,

India. 